\title{
Brain-derived neurotrophic factor in relation to central obesity in children with sleep disordered breathing
}

Karima Abdelfattah Bahgat ${ }^{1}$, Marwa Elhady ${ }^{1}$, Tawfik Abd Elatey Alkholy ${ }^{2}$, Fatma Mohamed Abd Elgaber ${ }^{2}$, Walaa Shipl ${ }^{3}$, Ayman A. Gobarah ${ }^{4}$

\begin{abstract}
${ }^{1}$ Department of Pediatrics, Faculty of Medicine, Al-Azhar University, Cairo, Egypt ${ }^{2}$ Faculty of Medicine, Al-Azhar University, Cairo, Egypt

${ }^{3}$ Department of Biochemistry, Faculty of Medicine, Al-Azhar University, Cairo, Egypt ${ }^{4}$ Department of Pediatrics, Faculty of Medicine, Suez Canal, Egypt
\end{abstract}

Submitted: 18 March 2018; Accepted: 21 August 2018

Online publication: 19 September 2019

Arch Med Sci 2021; 17 (5): 1332-1339

DOI: https://doi.org/10.5114/aoms.2019.86895

Copyright $\odot 2019$ Termedia \& Banach

\begin{abstract}
Introduction: Sleep disordered breathing (SDB) represents common comorbidities of childhood obesity leading to interrupted sleep and sleep deprivation. Sleep deprivation alters secretion of brain-derived neurotrophic factor (BDNF), which is an appetite regulator. However, little is known about the relation between BDNF and central obesity in children with SDB. The aim of the study was to evaluate BDNF level and anthropometric indices in relation to SDB in children with obesity

Material and methods: A prospective case-control study was conducted on 30 children with obesity (BMI $>95^{\text {th }}$ percentile) and 30 healthy lean children (BMI $5^{\text {th }}-85^{\text {th }}$ percentile). Polysomnographic, anthropometric data and BDNF serum level were obtained from all included children. Serum level of BDNF and anthropometric indices of obesity were assessed in relation to SDB in children with obesity. Regression analysis was done to determine predictors for SDB in children with obesity.

Results: In comparison to healthy controls, anthropometric indices of central obesity were significantly higher while BDNF was significantly lower in obese children, especially those with SDB. Respiratory disturbance index has a significant positive correlation with anthropometric indices of central obesity and a significant negative correlation with BDNF level. Central obesity and decreased BDNF were associated with 2-fold increased risk for SDB. Waist circumference/height ratio and neck circumference/height ratio have $89.5 \%, 75 \%$ sensitivity and $81.23 \%, 84.62 \%$ specificity at a cutoff point $>0.62,>0.24$ respectively for prediction of SDB in children with obesity. Conclusions: Central obesity and decreased BDNF represent independent predictors for SDB in children with obesity. Anthropometric indices adjusted to height are a simple screening tool for SDB in obese children.
\end{abstract}

Key words: sleep, brain-derived neurotrophic factor, obesity, children.

\section{Introduction}

Sleep is an important component of children's physical and intellectual health. Poor sleep in children is a serious health problem that should no longer be ignored. Untreated sleep-disordered breathing (SDB) leads to significant morbidity, which may affect neurocognitive development in children [1]. Sleep-disordered breathing is a group of sleep disorders

\author{
Corresponding author: \\ Marwa Elhady MD, PhD \\ Department of Pediatrics \\ Faculty of Medicine \\ Al-Azhar University \\ 1157 Cairo, Egypt \\ Phone: +20 1120977670 \\ E-mail: Marwaelhady93@ \\ yahoo.com, marwaelhady@ \\ azhar.edu.eg
}


characterized by recurrent, partial, or complete cessation of breathing that disrupts normal sleep that includes snoring, apneas, hypopneas and respiratory effort-related arousal. The prevalence of SDB in children ranged between 1.2 and $13.9 \%$. Diagnosis of SDB in children is difficult, requiring laboratory-based polysomnography, which is the gold standard for sleep assessment. Despite its advantages, polysomnography is expensive and gives information about sleep for a limited time [2].

Apnea is characterized by recurrent periods of cessation of breathing that could be central, obstructive or mixed according to the presence or absence of effort of breathing; while reduction in the amplitude of airflow is termed hypopnea [3]. Sleep-disordered breathing events are usually associated with sleep fragmentation, reduced oxygen saturation, and sympathetic nervous system activation [4]. The respiratory disturbance index (RDI) is a simple polysomnographic tool to assess SDB. RDI represent an indicator for respiratory event-related arousals that is calculated as the number of apneas, hypopneas, and respiratory effort related arousals per hour of sleep [5]. The underlying mechanism of obesity-related airflow disruption includes anatomic obstruction of the upper airway due to excess soft tissue bulk in the pharynx, changes in muscle tone and nerve activity during sleep [6].

Obesity-related comorbidities are linked to central obesity and are associated with higher fat deposition in the upper segment of the body than the lower segment [7, 8]. Body mass index (BMI) is the most commonly used anthropometric parameter for assessment of childhood obesity; however, it is not an accurate index of central obesity, which is the main risk factor for obesity-related comorbidities [9]. Other indices of central obesity such as waist circumference (WC) and hip circumference $(\mathrm{HC})$ are time-consuming, technically difficult, vary by eating and respiration and are affected by abdominal distention. Neck circumference is a simple, time-saving easy anthropometric parameter that reflects both central and upper body adiposity [10]. Adjustment of waist and neck circumference to height in children has emerged to eliminate the effect of continuous growth on the anthropometric measurements [11].

Brain-derived neurotrophic factor (BDNF) is a widely expressed neurotrophin in the brain that is involved in cognitive function, energy homeostasis, mood, and sleep regulation. BDNF has a critical role in hypothalamic regulation of food intake [12]. There is emerging evidence that sleep deprivation, especially of rapid eye movement sleep, alters the secretion of BDNF [13].

However, the relation between SDB and BDNF levels and central obesity is not completely clarified. We conducted this study to clarify the as- sociation between BDNF level and anthropometric indices in relation to SDB in children with obesity.

\section{Material and methods}

\section{Study population}

This case control prospective study was conducted on 60 children; 30 of them had obesity and 30 were lean according to World Health Organization (WHO) [14]. They were selected from the pediatric and otorhinolaryngology outpatient clinic of Alzahraa University hospital, Al-Azhar University, Cairo, Egypt during the period from April 2015 to September 2016. Informed written consent was obtained from parents of all children involved in the study in accordance to the guidelines of the local ethics committee of Al-Azhar University.

Over a 1-year period we evaluated 90 children with obesity but only 30 of them fulfilled our inclusion and exclusion criteria and agreed to undergo polysomnographic assessment. Inclusion criteria were $\mathrm{BMI}>95^{\text {th }}$ percentile for obese children and $\mathrm{BMI} 5^{\text {th }}-85^{\text {th }}$ percentile for healthy controls. Both genders were included. Their age range was 6-14 years. According to their polysomnographic findings, they were categorized into children with or without SDB.

The SDB group included children with partial, or complete cessation of breathing that disrupts normal sleep due to any of the following: snoring, apneas (either central or peripheral), hypopneas and respiratory effort-related arousal. All the previous sleep disorders were confirmed by polysomnography (PSG).

Children suffering from any acute, chronic medical disorder (e.g. metabolic, endocrine, cardiorespiratory, liver, renal diseases), genetic, neuromuscular disorder, skeletal malformation, adenotonsillar hypertrophy, or those who received medication that may affect their sleep habits were excluded from the study.

Control children were selected consecutively from age- and sex-matched healthy lean siblings of children with obesity. Their BMI values were $5^{\text {th }}-85^{\text {th }}$ percentile; they fulfilled the same exclusion criteria as children with obesity.

\section{Medical history and examination}

All children were subjected to full history taking including age, sex, medical, dietetic history, life style and sleep history and complete general and systemic examination including neurological, respiratory, cardiac, and ENT consultation.

\section{Anthropometric measurements}

Anthropometric measures were carefully measured including body weight (kilograms) while 
the child was in light clothes, height (centimeter) while the child had bare feet with straight knee, hip, waist and neck circumference (centimeters) using a flexible non-stretchable measuring tape. All parameters were recorded to the nearest 0.1 value. Body mass index was calculated as weight in kilograms divided by squared height in meters and was plotted by age and sex on the sex-specific growth chart. BMI was plotted on Egyptian growth charts [15]. Obesity was diagnosed if $\mathrm{BMI}$ is $\geq 95^{\text {th }}$ percentile for age and sex. $\mathrm{BMI} \geq 5^{\text {th }}$ but $<85^{\text {th }}$ percentile is considered normal [14]. Waist circumference (WC) was measured using a measuring tape over the unclothed abdomen, with measurements made halfway between the lower border of the ribs and the highest point of iliac crest in the standing position at the end of expiration [16]. Hip circumference (HC) was measured using a measuring tape at the maximum extension of the buttocks with the child standing erect [17]. Neck circumference (NC) was measured in horizontal plane at a point just below the thyroid cartilage and perpendicular to the long axis of the neck while the child looking straight ahead with shoulders down [18]. Neck circumference/ height $(\mathrm{NC} / \mathrm{Ht})$ ratio and waist circumference to height $(\mathrm{WC} / \mathrm{Ht})$ ratio were calculated. All clinical observations have been performed once in each patient/control.

\section{Polysomnography}

Overnight (at least 8 h) PSG for one night was carried out once among all included children using polysomnography (PSG) apparatus (model BWIII; Product of Neurovirtual USA) at the sleep laboratory of the pediatric neurophysiology unit at Alzahraa hospital, Al-Azhar University.

\section{Assessment of serum BDNF level}

Samples were collected on awaking while the patient was fasting after overnight PSG to avoid the effect of exercise and diet on BDNF. Two $\mathrm{ml}$ of venous blood was drawn and collected in serum separator tubes and centrifuged for serum separation. Serum was stored at $-20^{\circ} \mathrm{C}$ until the time for assessment. Steps of the assay were done according to the recommendations of the manufacturers. BDNF was measured in serum rather than plasma as serum measurements showed lower variability than EDTA-plasma measurements and was stable after $2 \mathrm{~h}$ storage at room temperature and after 6 months storage [19]. Determination of serum BDNF was performed using enzyme immunoassay for the in vitro quantitative measurement of human from Kamiya biomedical company (Cat. No. KT-8570). The normal range of serum BDNF is $1.5-30.9 \mathrm{ng} / \mathrm{ml}$ in healthy subjects [20].
The kit contains a pre-coated microtiter plate with anti-BDNF antibody. Before the assay, the standard was diluted 5 times by pipetting $50 \mu \mathrm{l}$ of standard dilution in each tube. Samples were also diluted 5 times by pipetting a sample dilution of $40 \mu \mathrm{l}$ to the testing sample well, then adding a testing sample of $10 \mu \mathrm{l}$ (sample final dilution is 5 -fold) then incubating for $30 \mathrm{~min}$ at $37^{\circ} \mathrm{C}$. Wash solution was diluted 30 -fold with distilled water. All samples were washed 5 times with washing solution then we added $50 \mu \mathrm{l}$ of HRP enzyme to each well except the blank, then incubated again for $30 \mathrm{~min}$ at $37^{\circ} \mathrm{C}$. All samples were washed again 5 times with washing solution. Chromogen solution $(100 \mu \mathrm{l})$ was added to each well then the reaction was stopped by adding stop solution $(50 \mu \mathrm{l})$ so the blue color changed to yellow. The blank well was considered as zero and the absorbance was read at $450 \mathrm{~nm}$ at 15 min from adding the stop solution. The enzyme-substrate reaction was terminated by the addition of a sulfuric acid solution and the color change was measured spectrophotometrically at a wavelength of $450 \mathrm{~nm} \pm 2 \mathrm{~nm}$. The concentration of BDNF in the samples was then determined by comparing the O.D. of the samples to the calibration curve. Each patient's blood test was measured once in a single well.

\section{Statistical analysis}

Statistical analysis was performed using SPSS (version 19.0; SPSS Inc., Chicago, IL, USA). Differences between groups were analyzed with the one-way ANOVA test. Further analysis between groups was done by post hoc multiple analysis Tukey test. Correlations between variables were performed using the Pearson correlation test for determination of the correlation coefficient $(r)$. Binary logistic regression analysis was done to determine the predictors of SDB in obese children. Receiver operating characteristic curves (ROC) were used to identify sensitivity and specificity and determine optimal cut-off points of anthropometric indices of central obesity for prediction of SDB in obese children. Sensitivity = true positive/ (true positive + false negative). Specificity = true negative / (true negative + false positive). $P$-value $<0.05$ was considered to be significant.

\section{Results}

This study included 30 children with obesity (10 males and 20 females); 16 of them (53.3\%) had SDB (12 had obstructive apnea, 4 had central apnea, 4 had mixed apnea, 14 had hypopnea, 9 had snoring, 10 had respiratory effort-related arousal). Most of them had more than one pattern of SDB. In addition, 30 healthy children served as a control group (14 male, 16 female). 
Table I. Comparison of anthropometric and polysomnographic data and serum level of brain derived neurotrophic factor among the studied children

\begin{tabular}{|c|c|c|c|c|c|}
\hline \multirow[t]{2}{*}{ Variables } & \multirow{2}{*}{$\begin{array}{c}\text { Obesity with } \\
\text { SDB } \\
(n=16)\end{array}$} & \multirow{2}{*}{$\begin{array}{c}\text { Obesity } \\
\text { without SDB } \\
(n=14)\end{array}$} & \multirow{2}{*}{$\begin{array}{l}\text { Healthy } \\
\text { children } \\
(n=30)\end{array}$} & \multicolumn{2}{|c|}{ ANOVA test/ $\chi^{2}$ test } \\
\hline & & & & $f / \chi^{2}$ & $P$-value \\
\hline Age [years] & $10.062 \pm 2.909$ & $10.571 \pm 2.623$ & $10.283 \pm 2.926$ & 0.119 & 0.888 \\
\hline $\begin{array}{l}\text { Gender } \\
\text { Male/female, } n(\%)\end{array}$ & $\begin{array}{l}5(31.2) / \\
11(68.8)\end{array}$ & $\begin{array}{l}5(35.7) / \\
9(64.3)\end{array}$ & $\begin{array}{l}14(46.6) / \\
16(53.4)\end{array}$ & 1.173 & 0.556 \\
\hline Weight [kg] & $60.26 \pm 16.08$ & $55.89 \pm 10.95$ & $34.97 \pm 10.43$ & 27.033 & $<0.0001^{*}$ \\
\hline Height $[\mathrm{cm}]$ & $137.25 \pm 13.14$ & $138.71 \pm 13.07$ & $137.37 \pm 13.82$ & 0.056 & 0.945 \\
\hline $\mathrm{BMI}\left[\mathrm{kg} / \mathrm{m}^{2}\right]$ & $31.97 \pm 2.57$ & $28.76 \pm 2.56$ & $18.98 \pm 2.71$ & 145.006 & $<0.0001^{\star}$ \\
\hline$W C[\mathrm{~cm}]$ & $94.19 \pm 4.79$ & $86.79 \pm 5.91$ & $65.84 \pm 12.30$ & 51.811 & $<0.0001^{\star}$ \\
\hline $\mathrm{WC} / \mathrm{Ht}$ ratio & $0.65 \pm 0.14$ & $0.61 \pm 0.09$ & $0.48 \pm 0.06$ & 31.227 & $<0.0001^{\star}$ \\
\hline $\mathrm{HC}[\mathrm{cm}]$ & $98.99 \pm 10.71$ & $105.78 \pm 13.46$ & $76.42 \pm 14.51$ & 28.186 & $<0.0001^{*}$ \\
\hline $\mathrm{NC}[\mathrm{cm}]$ & $35.68 \pm 1.37$ & $33.74 \pm 1.41$ & $29.33 \pm 3.07$ & 39.814 & $<0.0001^{\star}$ \\
\hline $\mathrm{NC} / \mathrm{Ht}$ ratio & $0.26 \pm 0.02$ & $0.24 \pm 0.02$ & $0.21 \pm 0.02$ & 32.126 & $<0.0001^{\star}$ \\
\hline $\mathrm{O}_{2}$ minimum & $86.50 \pm 2.61$ & $95.00 \pm 1.62$ & $94.37 \pm 2.07$ & 82.565 & $<0.0001^{*}$ \\
\hline SE \% & $86.16 \pm 4.10$ & $90.80 \pm 2.90$ & $93.89 \pm 2.23$ & 34.923 & $<0.0001^{\star}$ \\
\hline NREM $1 \%$ & $9.66 \pm 2.49$ & $7.22 \pm 2.44$ & $4.88 \pm 0.83$ & 36.262 & $<0.0001^{\star}$ \\
\hline NREM $2 \%$ & $50.11 \pm 1.82$ & $47.85 \pm 2.65$ & $49.53 \pm 2.76$ & 3.198 & $0.048^{*}$ \\
\hline NREM3\% & $19.06 \pm 2.05$ & $22.65 \pm 2.19$ & $22.51 \pm 2.01$ & 16.602 & $<0.0001^{\star}$ \\
\hline REM\% & $20.15 \pm 1.76$ & $22.71 \pm 2.61$ & $23.13 \pm 2.07$ & 10.534 & $<0.0001^{*}$ \\
\hline $\mathrm{Al}$ & $10.92 \pm 3.61$ & $6.52 \pm 2.10$ & $2.79 \pm 2.66$ & 42.763 & $<0.0001^{\star}$ \\
\hline Sleep latency [min] & $10.06 \pm 3.68$ & $8.64 \pm 3.34$ & $11.70 \pm 2.98$ & 4.911 & $0.011^{*}$ \\
\hline REM latency [min] & $109.19 \pm 8.36$ & $106.43 \pm 6.62$ & $100.63 \pm 10.50$ & 4.378 & $0.017^{*}$ \\
\hline $\mathrm{BDNF}[\mathrm{ng} / \mathrm{ml}]$ & $0.86 \pm 0.47$ & $2.94 \pm 0.67$ & $3.86 \pm 1.25$ & 48.054 & $<0.0001^{*}$ \\
\hline \multicolumn{6}{|c|}{ Post hoc: multiple analysis } \\
\hline Variables & \multicolumn{2}{|c|}{$\begin{array}{l}\text { Obesity with SDB vs. } \\
\text { obesity without SDB }\end{array}$} & $\begin{array}{l}\text { Obesity with SDB vs. } \\
\text { healthy children }\end{array}$ & Obes & $\begin{array}{l}\text { out SDB vs. } \\
\text { children }\end{array}$ \\
\hline Weight [kg] & \multicolumn{2}{|c|}{0.261} & $<0.0001^{*}$ & \multicolumn{2}{|c|}{$<0.0001^{*}$} \\
\hline BMI $\left[\mathrm{kg} / \mathrm{m}^{2}\right]$ & \multicolumn{2}{|c|}{$0.001^{*}$} & $<0.0001^{*}$ & \multicolumn{2}{|c|}{$<0.0001^{*}$} \\
\hline$W C[\mathrm{~cm}]$ & \multicolumn{2}{|c|}{$0.023^{*}$} & $<0.0001^{*}$ & \multicolumn{2}{|c|}{$<0.0001^{*}$} \\
\hline $\mathrm{WC} / \mathrm{Ht}$ ratio & \multicolumn{2}{|c|}{$0.008^{*}$} & $<0.0001^{*}$ & \multicolumn{2}{|c|}{$<0.0001^{*}$} \\
\hline $\mathrm{HC}[\mathrm{cm}]$ & \multicolumn{2}{|c|}{0.224} & $<0.0001^{*}$ & \multicolumn{2}{|c|}{$<0.0001^{*}$} \\
\hline $\mathrm{NC}[\mathrm{cm}]$ & \multicolumn{2}{|c|}{$0.026^{*}$} & $<0.0001^{*}$ & \multicolumn{2}{|c|}{$<0.0001^{*}$} \\
\hline $\mathrm{NC} / \mathrm{Ht}$ ratio & \multicolumn{2}{|c|}{$0.002^{*}$} & $<0.0001^{*}$ & \multicolumn{2}{|c|}{$<0.0001^{*}$} \\
\hline $\mathrm{O}_{2}$ minimum & \multicolumn{2}{|c|}{$<0.0001^{\star}$} & $<0.0001^{*}$ & \multicolumn{2}{|c|}{0.368} \\
\hline SE \% & \multicolumn{2}{|c|}{$<0.0001^{*}$} & $<0.0001^{*}$ & \multicolumn{2}{|c|}{$0.001^{*}$} \\
\hline NREM $1 \%$ & 0.014 & & 0.531 & & \\
\hline NREM $2 \%$ & $<0.000$ & & $<0.0001^{*}$ & & \\
\hline NREM3\% & 0.001 & & $<0.0001^{*}$ & & \\
\hline REM\% & $0.50 s$ & & $0.022^{*}$ & & \\
\hline $\mathrm{Al}$ & 0.26 & & $0.002^{*}$ & & \\
\hline RDI: & & & & & \\
\hline Sleep latency [min] & $<0.000$ & & $<0.0001^{*}$ & & \\
\hline REM latency [min] & $<0.000$ & & $<0.0001^{*}$ & & $001^{*}$ \\
\hline $\mathrm{BDNF}[\mathrm{ng} / \mathrm{ml}]$ & $<0.000$ & & $<0.0001^{*}$ & & \\
\hline
\end{tabular}

${ }^{*}$ Significant. BMI - body mass index, WC - waist circumference, WC/Ht - waist circumference/height, $H C$ - hip circumference, NC - neck circumference, NC/Ht - neck circumference/height, SE - sleep efficiency, Al - arousal index, RDI - respiratory disturbance index, NREM non-rapid eye movement, REM - rapid eye movement, BDNF-brain-derived neurotrophic factor. 


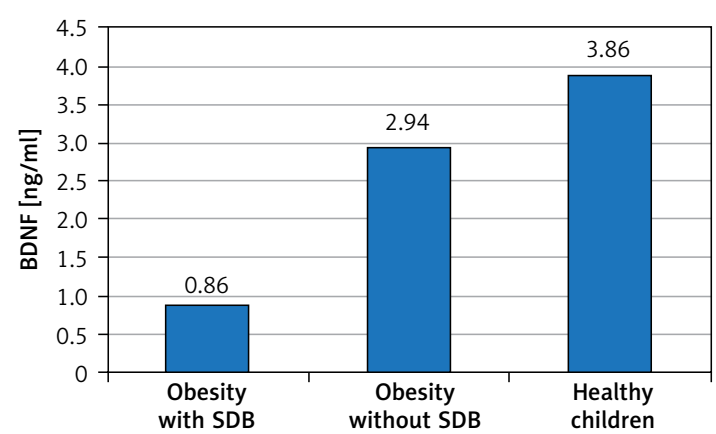

Figure 1. Comparison of serum BDNF among the studied children

We evaluated serum level of BDNF and anthropometric parameters as independent variables in relation to SDB detected by PSG as a dependent variable in the studied children. Table I and Figure 1 show the comparison of anthropometric parameters, polysomnographic findings, and BDNF serum level of the included children. No substantial age and gender differences were found among children involved in the study. BDNF was significantly lower in obese children especially those with SDB. Further analysis between subgroups revealed that obese children with SDB had statistically significantly higher indices of central obesity (WC, $\mathrm{NC}, \mathrm{WC} / \mathrm{Ht}$ ratio, NC/Ht ratio) and a statistically significantly lower BDNF serum level than those without SDB and healthy controls. WC that represents an index of peripheral obesity did not show a significant difference between those with and without SDB. As regard polysomnographic findings, obese children had statistically significantly more disturbed sleep and lower sleep ef-

Table II. Correlation between anthropometric parameters, serum level of brain-derived neurotrophic factor and polysomnographic data in children with obesity

\begin{tabular}{|lcc|}
\hline Variables & \multicolumn{2}{c|}{ RDI } \\
\cline { 2 - 3 } & \multicolumn{2}{c|}{ P-value } \\
\hline Weight $[\mathrm{kg}]$ & 0.085 & 0.654 \\
\hline BMI $\left[\mathrm{kg} / \mathrm{m}^{2}\right]$ & 0.613 & $<0.0001^{*}$ \\
\hline WC $[\mathrm{cm}]$ & 0.513 & $0.004^{*}$ \\
\hline WC/Ht ratio & 0.638 & $<0.0001^{*}$ \\
\hline HC $[\mathrm{cm}]$ & 0.421 & $0.020^{*}$ \\
\hline NC $[\mathrm{cm}]$ & 0.509 & $0.004^{*}$ \\
\hline NC/Ht ratio & 0.638 & $<0.0001^{*}$ \\
\hline BDNF [ng/ml] & -0.765 & $<0.0001^{*}$ \\
\hline
\end{tabular}

*Significant. BMI - body mass index, WC - waist circumference, WC/Ht - waist circumference/height, HC - hip circumference, NC - neck circumference, SE - sleep efficiency, Al - arousal index, $\mathrm{RDI}$ - respiratory disturbance index, $\mathrm{NC} / \mathrm{Ht}$ - neck circumferencel height, BDNF- brain-derived neurotrophic factor. ficiency especially those with SDB than healthy controls.

Pearson's correlation test was used to assess the correlations between RDI as an indicator of SDB and anthropometric parameters. Table II and Figure 2 show a significant negative correlation between RDI and BDNF serum level and a significant positive correlation between RDI and anthropometric indices of obesity (BMI, WC, WC/Ht ratio, $\mathrm{HC}, \mathrm{NC}, \mathrm{NC} / \mathrm{Ht}$ ratio).

Logistic regression analysis showed that anthropometric indices of central obesity and low BDNF were risk factors for SDB in obese children as shown in Table III.

A ROC curve was plotted according to the patient data. Waist circumference/height ratio and neck circumference/height ratio have $89.5 \%, 75 \%$ sensitivity and $81.23 \%, 84.62 \%$ specificity at a cutoff point $>0.62,>0.24$ respectively for prediction of SDB in obese children as shown in Table IV.

\section{Discussion}

Obesity is a major risk factor for SDB that adversely affects the sleep efficiency and duration and further increases the risk for more weight gain [21]. BDNF is an important neurotransmitter that regulates both sleep and appetite [22], but its role as a link between obesity and SDB was not well explored in children.

Among our studied children, SDB was detected in $53.3 \%$ of children with obesity. This agrees with previous reports that obesity is a major risk factor for sleep apnea in both children [23] and adults [24]. Verhulst et al. [25] reported that $47 \%$ of children with obesity had SDB. There is $12 \%$ increased risk of obstructive sleep apnea for every one unit increase in BMI [26] and a 6-fold increase in the risk of SDB for every $10 \%$ increase in body weight [27].

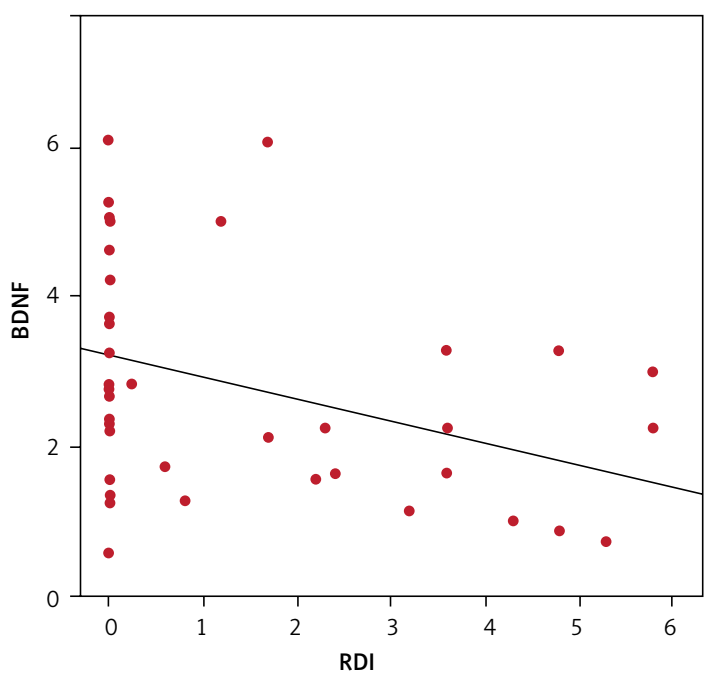

Figure 2. Correlation between serum level of BDNF and RDI in the studied children 
Table III. Logistic regression analysis of predictors of sleep disordered breathing in children with obesity

\begin{tabular}{|c|c|c|c|c|}
\hline \multirow[t]{2}{*}{ Variables } & \multirow[t]{2}{*}{ OR } & \multicolumn{2}{|c|}{$95 \% \mathrm{Cl}$ for $\mathrm{OR}$} & \multirow[t]{2}{*}{$P$-value } \\
\hline & & Lower & Upper & \\
\hline Weight [kg] & 1.003 & 0.991 & 1.015 & 0.579 \\
\hline $\mathrm{BMI}\left[\mathrm{kg} / \mathrm{m}^{2}\right]$ & 2.008 & 0.984 & 2.932 & $0.038^{*}$ \\
\hline$W C[\mathrm{~cm}]$ & 2.002 & 0.994 & 3.010 & $0.041^{*}$ \\
\hline $\mathrm{WC} / \mathrm{Ht}$ ratio & 3.381 & 1.136 & 6.694 & $0.012^{*}$ \\
\hline $\mathrm{HC}[\mathrm{cm}]$ & 1.001 & 0.994 & 1.008 & 0.855 \\
\hline $\mathrm{NC}[\mathrm{cm}]$ & 1.005 & 0.985 & 1.026 & 0.106 \\
\hline $\mathrm{NC} / \mathrm{Ht}$ ratio & 2.358 & 1.002 & 5.842 & $0.035^{*}$ \\
\hline $\mathrm{BDNF}[\mathrm{ng} / \mathrm{ml}]$ & 3.115 & 0.838 & 5.944 & $0.017^{*}$ \\
\hline
\end{tabular}

Table IV. Sensitivity, specificity, and predictive values for diagnosis of sleep disordered breathing using anthropometric indices of central obesity

\begin{tabular}{|lcccccc|}
\hline Variables & Cut-off & AUC & Sensitivity (\%) & Specificity (\%) & +PV & -PV \\
\hline BMI $\left[\mathrm{kg} / \mathrm{m}^{2}\right]$ & $>30.02$ & 0.827 & 81.25 & 76.92 & 28.1 & 97.4 \\
\hline WC $[\mathrm{cm}]$ & $>88.3$ & 0.875 & 87.5 & 76.92 & 29.6 & 98.2 \\
\hline WC/Ht ratio & $>0.62$ & 0.884 & 89.5 & 81.23 & 32.4 & 98.8 \\
\hline NC $[\mathrm{cm}]$ & $>34.3$ & 0.853 & 87.5 & 69.23 & 24 & 98 \\
\hline NC/Ht ratio & $>0.24$ & 0.834 & 75 & 84.62 & 35.1 & 96.8 \\
\hline
\end{tabular}

$B M I$ - body mass index, WC - waist circumference, WC/Ht - waist circumference/height, NC - neck circumference, NC/Ht - neck circumference/height, $+P V$ - positive predictive value, $-P V$ - negative predictive value.

Polysomnography is the gold standard for diagnosis of SDB in children. However, simple screening tools are needed for high risk populations. Our study demonstrated significant association between SDB, BMI and other anthropometric indices of central obesity including WC/Ht and NC/ $\mathrm{Ht}$ ratio. WC/Ht ratio has the highest sensitivity (89.5\%) while NC/Ht ratio has the highest specificity (84.6\%) for prediction of SDB in obese children. The significant correlation between increased WC, NC, BMI and SDB was reported even in general populations [28]. Previous evidence revealed significant association between SDB and central obesity [29]. However there were controversial reports regarding the best anthropometric parameter to predict SDB in obese subjects [30-32].

To overcome the variation in anthropometry in relation to ages of included children we use WC/ $\mathrm{Ht}$ and $\mathrm{NC} / \mathrm{Ht}$ ratio as indicators of central obesity. Our study revealed that obese children with SDB have higher $\mathrm{WC} / \mathrm{Ht}$ and $\mathrm{NC} / \mathrm{Ht}$ ratio than those without SDB or healthy controls. Additionally, regression analysis of risk factors for SDB in our studied children revealed that $\mathrm{WC} / \mathrm{Ht}$ and $\mathrm{NC} / \mathrm{Ht}$ ratio were strong predictors for SDB. Similar findings were reported by Ho et al. [33], who conclud- ed that a cutoff point $>0.25 \mathrm{NC} / \mathrm{Ht}$ ratio is a valuable screening tool for obstructive sleep apnea in both children and adults.

Regarding the impact of obesity on polysomnographic findings, our study revealed that obese children with SDB have more disturbed sleep architecture, poor sleep efficiency and lower oxygen saturation than those without SDB and healthy controls. Jarrin et al. [34] reported poorer sleep quality and more sleep disturbances in obese children. Chamorro et al. [35] found that sleep efficiency was inversely associated with BMI. Peppard et al. [36] found that obesity exaggerates the oxygen desaturation during SDB with a $1 \%$ increase in oxygen desaturation for each $10 \mathrm{~kg} / \mathrm{m}^{2}$ increase of BMI.

Sleep disorders may be related to a combination of biological, psychological, and anatomical factors. Several mechanisms are suggested to explain the associations between obesity and SDB, including low upper airway muscle tone, and airway collapsibility due to increased parapharyngeal tissue fat content leading to activation of different exaggerated forms of trigemino-cardiac reflex. Trigeminal stimulation during sleep leads to arousals in a dose- and time-dependent manner 
[37]. Other mechanisms include altered chest-wall mechanics, reduced lung volume causing lower oxygen reserves, as well as impairment of ventilatory control induced by leptin and cytokines. Reduction in oxygen saturation due to frequent SDB leads to cortical and brainstem arousals and disturbed sleep. There is evidence that weight loss can reduce SDB in obese patients [38].

Children with obesity are more vulnerable to sleep fragmentation and arousal induced by respiratory events leading to short sleep durations and chronic sleep deprivation [39, 40]. Some evidence showed a strong association between poor sleep and obesogenic eating behavior; however, the underlying mechanism is not well understood [41].

Our results demonstrated a significant decrease of peripheral BDNF in obese children either with or without SDB. BDNF plays an important role in regulation of sleep and appetite. Short sleep deprivations increase cortical expression of BDNF but chronic prolonged sleep deprivations decrease it [42]. A decreased level of BDNF places more burden on children with obesity. A previous report suggested that serum BDNF was inversely related to appetite [43].

In our study, children with obesity had a significant higher arousal index than healthy children reflecting interrupted sleep and poor sleep quality, which could explain the lower serum level of BDNF in children with obesity, particularly those with SDB. These findings are in accordance with accumulated evidence linking sleep deprivation to decreased BDNF [44]. Wang et al. [45] reported a significantly reduced BDNF serum level in patients with obstructive sleep apnea which was related to nocturnal hypoxia as well and deprivation of slow wave sleep and REM sleep in such patients.

One of the major limitations of our study is the small number of included children. The diagnosis of central obesity was done on the basis of anthropometry instead of dual-energy X-ray absorptiometry, which is the gold standard for assessment of visceral obesity. Previous evidence demonstrated the validity of simple anthropometric parameters for assessment of central obesity [46].

Additionally, the central level of BDNF could not be measured; however, a previous report [47] demonstrated that BDNF can readily cross the blood-brain barrier, suggesting that the peripheral level of BDNF could reflect its brain expression.

In conclusion, children with obesity have a decreased BDNF serum level. Central obesity and decreased BDNF represent independent predictors for SDB in children with obesity. Anthropometric indices of central obesity adjusted to height can be used as a simple screening tool for SDB in children. Further studies are required to illustrate the effect of weight reduction on BDNF serum level and to assess the potential therapeutic benefit of BDNF on SDB among children with obesity.

\section{Conflict of interest}

The authors declare no conflict of interest.

\section{References}

1. Gregory AM, Sadeh A. Annual research review: sleep problems in childhood psychiatric disorders - a review of the latest science. J Child Psychol Psychiatry 2016; 57: 296-317.

2. Bixler EO, Vgontzas AN, Lin HM, et al. Sleep disordered breathing in children in a general population sample: prevalence and risk factors. Sleep. 2009; 32: 731-6.

3. Dempsey JA, Veasey SC, Morgan BJ, et al. Pathophysiology of sleep apnea. Physiol Rev 2010; 90: 47-112.

4. Pham LV, Schwartz AR. The pathogenesis of obstructive sleep apnea. J Thorac Dis 2015; 7: 1358-72.

5. Richardson, Mark A., Friedman, Norman R (eds.). Clinician's Guide to Pediatric Sleep Disorders, p. 75. Informa Healthcare USA, New York 2007.

6. Narang I, Mathew JL. Childhood obesity and obstructive sleep apnea. J Nutr Metab 2012; 2012: 134202.

7. Papaetis GS, Papakyriakou P, Panagiotou TN. Central obesity, type 2 diabetes and insulin: exploring a pathway full of thorns. Arch Med Sci 2015; 11: 463-82.

8. Jelavic MM, Babic Z, Pintaric $H$. The importance of two metabolic syndrome diagnostic criteria and body fat distribution in predicting clinical severity and prognosis of acute myocardial infarction. Arch Med Sci 2017; 13: 795-806.

9. Cheong KC, Ghazali SM, Hock LK, et al. The discriminative ability of waist circumference, body mass index and waist-to-hip ratio in identifying metabolic syndrome: variations by age, sex and race. Diabetes Metab Syndr 2015; 9: 74-8.

10. Taheri M, Kajbaf TZ, Taheri MR, Aminzadeh M. Neck circumference as a useful marker for screening overweight and obesity in children and adolescents. Oman Med J 2016; 31: 170-5.

11. Selvan C, Dutta D, Thukral A, et al. Neck height ratio is an important predictor of metabolic syndrome among Asian Indians. Indian J Endocrinol Metab 2016; 20: 831-7.

12. Bathina S, Das UN. Brain-derived neurotrophic factor and its clinical implications. Arch Med Sci 2015; 11: 1164-78.

13. Zielinski MR, Kim Y, Karpova SA, et al. Chronic sleep restriction elevates brain interleukin-1 beta and tumor necrosis factor-alpha and attenuates brain-derived neurotrophic factor expression. Neurosci Lett 2014; 580: 27-31.

14. WHO. Global status report on noncommunicable diseases 2014. Chapter 7: 79-94.

15. Ghali I, Salah N, Hussien F, et al. Egyptian Growth Curves 2002 for Infants, Children and Adolescents. Ferring Publisher, Crescere nel mondo 2008.

16. World Health Organization. WHO STEP wise approach to surveillance (STEPS): Guide to physical measurements. World Health Organization; 2008. Available at: http://www.who.int/chp/steps/Part3_Section3.pdf.

17. Kuk JL, Janiszewski PM, Ross R. Body mass index and hip and thigh circumferences are negatively associated with visceral adipose tissue after control for waist circumference. Am J Clin Nutr 2007; 85: 1540-4. 
18. Hingorjo MR, Qureshi MA, Mehdi A. Neck circumference as a useful marker of obesity: a comparison with body mass index and waist circumference. J Pak Med Assoc 2012; 62: 36-40.

19. Polyakova M, Schlögl $H$, Sacher J, et al. Stability of BDNF in human samples stored up to 6 months and correlations of serum and EDTA-plasma concentrations. Int J Mol Sci 2017; 18: 1189.

20. Knaepen K, Goekint M, Heyman EM, et al. Neuroplasticity - exercise-induced response of peripheral brain-derived neurotrophic factor: a systematic review of experimental studies in human subjects. Sports Med 2010; 40: 765-801.

21. Leinum CJ, Dopp JM, Morgan BJ. Sleep disordered breathing and obesity: pathophysiology, complications and treatment. Nutr Clin Pract 2009; 24: 675-87.

22. Hempstead BL. Brain-derived neurotrophic factor: three ligands, many actions. Trans Am Clin Climatol Assoc 2015; 126: 9-19.

23. Ma Y, Peng L, Kou C, Hua S, Yuan H. Associations of overweight, obesity and related factors with sleep-related breathing disorders and snoring in adolescents: a cross-sectional survey. Int J Environ Res Public Health 2017; 14: 194.

24. Peppard PE, Young T, Barnet JH, Palta M, Hagen EW, Hla KM. Increased prevalence of sleep-disordered breathing in adults. Am J Epidemiol 2013; 177: 1006-14.

25. Verhulst SL, Schrauwen N, Haentjens D, et al. Sleep-disordered breathing in overweight and obese children and adolescents: prevalence, characteristics and the role of fat distribution. Arch Dis Child 2007; 92: 205-8.

26. Narang I, Mathew JL. Childhood obesity and obstructive sleep apnea. J Nutr Metab 2012; 2012: 134202.

27. Peppard PE, Young T, Palta M, Dempsey J, Skatrud J. Longitudinal study of moderate weight change and sleep-disordered breathing. JAMA 2000; 284: 3015-21.

28. Kang HH, Kang JY, Ha JH, et al. The associations between anthropometric indices and obstructive sleep apnea in a Korean population. PLoS One 2014; 9: e114463.

29. Nafiu OO, Prasad Y, Chimbira WT. Association of childhood high body mass index and sleep disordered breathing with perioperative laryngospasm. Int J Pediatr Otorhinolaryngol 2013; 77: 2044-8.

30. Davidson TM, Patel MR. Waist circumference and sleep disordered breathing. Laryngoscope 2008; 118: 339-47.

31. Ahbab S, Ataoğlu HE, Tuna M, et al. Neck circumference, metabolic syndrome and obstructive sleep apnea syndrome; evaluation of possible linkage. Med Sci Monit 2013; 19: 111-7.

32. Onat A, Hergenç G, Yüksel $H$, et al. Neck circumference as a measure of central obesity: associations with metabolic syndrome and obstructive sleep apnea syndrome beyond waist circumference. Clin Nutr 2009; 28: 46-51.

33. Ho AW, Moul DE, Krishna J. Neck circumference-height ratio as a predictor of sleep related breathing disorder in children and adults. J Clin Sleep Med 2016; 12: 311-7.

34. Jarrin DC, McGrath JJ, Drake CL. Beyond sleep duration: distinct sleep dimensions are associated with obesity in children and adolescent's. Int J Obes (Lond) 2013; 37: 552-8.

35. Chamorro R, Algarín C, Garrido M, et al. Nighttime sleep macrostructure is altered in otherwise healthy 10-yearold overweight children. Int J Obes (Lond) 2014; 38 1120-5.

36. Peppard PE, Ward NR, Morrell MJ. The impact of obesity on oxygen desaturation during sleep-disordered breathing. Am J Respir Crit Care Med 2009; 180: 788-93.
37. Chowdhury T, Bindu B, Singh GP, et al. Sleep disorders: is the trigemino-cardiac reflex a missing link? Front Neurol 2017; 8: 63.

38. Shapiro SD, Chin CH, Kirkness JP, et al. Leptin and the control of pharyngeal patency during sleep in severe obesity. J Appl Physiol (1985) 2014; 116: 1334-41.

39. Miller AL, Lumeng JC, LeBourgeois MK. Sleep patterns and obesity in childhood. Curr Opin Endocrinol Diabetes Obes 2015; 22: 41-7.

40. Ruchała M, Bromińska B, Cyrańska-Chyrek E, Kuźnar-Kamińska B, Kostrzewska M, Batura-Gabryel H. Obstructive sleep apnea and hormones - a novel insight. Arch Med Sci 2017; 13: 875-84.

41. Spaeth AM, Dinges DF, Goel N. Effects of experimental sleep restriction on weight gain, caloric intake, and meal timing in healthy adults. Sleep 2013; 36: 981-90.

42. Schmitt K, Holsboer-Trachsler E, Eckert A. BDNF in sleep, insomnia, and sleep deprivation. Ann Med 2016; 48: 42-51.

43. Stanek K, Gunstad J, Leahey T, et al. Serum brain-derived neurotrophic factor is associated with reduced appetite in healthy older adults. J Nutr Health Aging 2008; 12 : 183-5.

44. Saito S, Watanabe K, Hashimoto E, Saito T. Low serum BDNF and food intake regulation: a possible new explanation of the pathophysiology of eating disorders. Prog Neuropsychopharmacol Biol Psychiatry 2009; 33: 312-6.

45. Wang WH, He GP, Xiao XP, Gu C, Chen HY. Relationship between brain-derived neurotrophic factor and cognitive function of obstructive sleep apnea/hypopnea syndrome patients. Asian Pac J Trop Med 2012; 5: 906-10.

46. Sun Q, van Dam RM, Spiegelman D, Heymsfield SB, Willett WC, Hu FB. Comparison of dual-energy X-ray absorptiometric and anthropometric measures of adiposity in relation to adiposity-related biologic factors. Am J Epidemiol 2010; 172: 1442-54.

47. Pan W, Banks WA, Fasold MB, Bluth J, Kastin AJ. Transport of brain-derived neurotrophic factor across the blood-brain barrier. Neuropharmacology 1998; 37: 1553-61. 